\title{
Break the Germ Cycle: Hand Hygiene Compliance among Healthcare Workers in an Accredited Tertiary Care Hospital
}

\author{
Article By Jessy Sebastian \\ MSN, Texila American University, United Arab Emirates \\ Email: Jessysebastian4@Yahoo.Com
}

\begin{abstract}
Health care associated infections affect hundreds of millions of people around the world and it is a major global issue for patient safety. Moreover, the most common way that micro-organisms (germs), particularly bacteria, are spread and cause infection is by being carried on people's hands. Hand hygiene is the most important measure to avoid the spread of harmful germs and to prevent ill health. Regular and thorough hand hygiene is always important when working in an environment or organization where health care is provided. So, having clean hands helps to protect patients, particularly the most vulnerable, as well as health care workers.

This change project proposed to improve hand hygiene compliance among health care workers in Intensive care units (Cardiac, Medical \& Neonatal ICU's) of a tertiary hospital in UAE. This included increasing compliance to hand washing, as well as introducing another simple, quick and effective procedure of hand hygiene which is using alcohol hand rub. The hand hygiene compliance rate showed gradual improvement over time, starting from below standard hand hygiene at base line assessment (44\%), to reach its maximum at 3 weeks post intervention assessment (94\%). These results indicate that the systematic application of the change model and WHO multimodal strategies is associated with improvement in hand hygiene compliance of healthcare workers and thus it could result in a sustained positive impact.

Although hand washing was found to be the preferred method for hand hygiene at 3 weeks into the intervention, yet the preference was reversed in the 3 weeks post intervention assessment to alcohol hand rub. In general, the literature suggests healthcare workers are possibly more concerned about their own safety than transmitting infection to patient. Yet in this project, they are found to be in different to the two indications with similar levels of compliance at baseline and postintervention.
\end{abstract}

\section{Introduction}

\section{Background of study}

Hand hygiene (i.e., washing hands with soap and water, or disinfection using alcohol- based hand rub) remains the single most important measure of preventing the spread of antimicrobial resistant pathogens and subsequent nosocomial infection. (Barrs A, 2000 and Pittet. et al 2006). Hand washing or hand hygiene is an ancient cultural custom that goes back to an immemorial time. It was observed primarily to remove dirt and to relieve people symbolically from physical and moral evils.

Barbacombe (2004) suggests -it is so basic, so simple, almost insulting or embarrassing even to mention, especially at an advanced practice level. Unfortunately, in health care, compliance with hand hygiene practices has been below an acceptable level. One study aimed to measure medical staff attitude towards hand hygiene; this showed compliance rates of hand cleaning of less than $50 \%$ (Bischoff, Reynolds, Scssler, Edmond and Wenzel, 2000). Another study done on a similar topic showed a compliance rate of $63 \%$. (Suchitra \& Lakshmidevi, 2006). These studies demonstrate that hand hygiene practices are below an acceptable level. So failure to perform appropriate hand hygiene either by washing or disinfection by any means or technique is considered to be the leading cause of infection. 
Every day, consciously and unconsciously, we make decisions regarding our patients' care. To make clinical decisions, almost instinctively we resort to variety of resources, including our own clinical experiences and discussions with colleagues - we rely on textbooks, journal articles, and previous educational experiences. As educators, we should evaluate the methodology of teaching students and residents how to make clinical decisions. Evidence-based practice (EBP) is an approach to health care wherein health professionals use the best evidence possible, i.e. The most appropriate information available, to make clinical decisions for a patient. EBP values, enhances, and builds on clinical expertise, knowledge of disease mechanisms, and patho-physiology (Macnee, 2004).

Over the past few years, scientific evidence to support the role of hand hygiene in the improvement of patient safety has increased considerably, but some key controversial issues still challenge care practitioners and researchers. Key among these is the question of compliance in practice and it is now recognized that there is no single solution; rather multimodal programmes are needed to promote compliance (Pittet D et al, 2000).

\section{Objective of the study}

-To evaluate compliance with the opportunity of hand hygiene by health care personnel within 3 Intensive Care Units in my Hospital, following the implementation of WHO Hand Hygiene Implementation multimodal improvement strategy and applying the HSE change model

- To understand the behavioral determinants of hand hygiene in the 3 Intensive Care Units

- To assess the health care workers (HCW) knowledge \& and perception/attitude change in response to the implementation multimodal improvement strategy namely the hand hygiene training program

\section{Significance of the study}

Health care associated infections affect hundreds of millions of people around the world and it is a major global issue for patient safety (WHO, 2009). In general, infections have a multifactorial causation related to systems and processes of health care provision as well as to political and economic constraints on health systems (WHO, 2009). Moreover, the most common way that microorganisms (germs), particularly bacteria, are spread and cause infection is by being carried on people's hands (CDC, 2002).

Hand hygiene is the most important measure to avoid the spread of harmful germs and to prevent ill health (Pittet, Allegranzi \& Storr, 2008). Regular and thorough hand hygiene is always important when working in an environment or organisation where health care is provided. So, having clean hands helps to protect patients, particularly the most vulnerable, as well as protecting health care workers (WHO, 2005).

\section{Research Questions}

1. Does implementation of WHO Hand Hygiene Implementation Multi-modal improvement strategy increasethe compliance of Hand Hygiene?

2. Will applying the HSE change modeldecrease Health care associated infections?

\section{Scope of study}

This quality improvement project aimed to remove the Hawthorne effect and improve the behavior of Healthcare workers in Intensive Care Units in a tertiary Hospital.

\section{Limitation}

The limitations of this project were based on the health care workers performing Hand hygiene. The study mainly focussed on the assessment of the behaviour of the health care workers towards hand hygiene. 


\section{Literature Review}

\section{Introduction}

There is a wealth of literature reviewed in this chapter that supports the importance of hand hygiene to preventing healthcare associated infections (HCAIs). The impact of these nosocomial infections is both a direct risk to patients and also increases the service and financial burden to health systems. As a significant safety hazard, multimodal strategies for hand hygiene have been developed as an evidence based global response. In addition, this chapter considers hand hygiene from the historical and cultural perspectives, as well as the main factors affecting compliance.

\section{Method of literature review}

Searches of the literature were conducted using RCSI library resources. I mainly used Science direct and Medline. Other resources include Google scholar, but most of the useful research articles found were not accessible. Also I was lucky enough to have the password for HINARI which is a good web link offered by the World Health Organization that gives access to most of the international journals.

The search terms used were: - healthcare associated infection - hand hygiene compliance - hand hygiene and compliance - hospital acquired infection - hand washing compliance - hand washing and compliance - hand disinfection compliance - hand rub compliance-hand decontamination compliance.

The related articles for each search were reviewed according its relevance. The criteria of selection included: the original research article or a systematic review article, studies exploring compliance of hand hygiene among health care workers and the language in English. Relevant papers were selected by screening the title (first step), the abstracts (second step), then the entire articles (third step) retrieved during the database searches.

\section{Healthcare-Associated Infections:}

Healthcare-Associated Infections (HCAIs) represent a major risk to patient safety and contribute towards suffering, prolongation of hospital stay, cost and mortality (Cosgrove, 2006 \& Graves, Weinhold and Tong E, et al 2007). In addition to that, the impact of HCAIs implies long-term disability, increases resistance of microorganisms to antimicrobials, adds massive additional financial burdens, increases fatalities, poses a high costs for the health systems, let alone the emotional stress for patients and their families.

Although the risk of acquiring HCAI is universal and pervades every health-care facility and system around the world, the global burden is unknown because of the difficulty of gathering reliable diagnostic data (WHO, 2009). Overall estimates indicate that more than 1.4 million patients worldwide in developed and developing countries are affected at any time. (World Alliance for Patient Safety, 2005).

In developed countries, HCAI concerns $5-15 \%$ of hospitalized patients and can affect $9-37 \%$ of those admitted to intensive care units. (WHO, 2005 \& Vincent, 2003). While in developing countries, many additional factors contribute to increase the risk of HCAI, namely: poor hygiene and sanitation, lack or shortage of basic equipment, and inadequate structures and overcrowding, an unfavorable social background and a population largely affected by malnutrition and other types of infection and/or diseases (Ofner-Agostini et al., 2006 ) \& Ho PL, Tang XP, Seto WH., 2003).

Limited data on HCAI from these developing country settings are available from the literature (WHO, 2009). In addition to the usual difficulties to define the diagnosis of HCAI, there is also paucity and unreliability of laboratory data, lack of standardized information from medical records, and scarce access to radiological facilities. The costs of managing HCAI are likely to represent a higher percentage of the health or hospital budget in low income countries as well.

Healthcare workers (HCWs) can also become infected during patient care as the risk of transmission is two-way. During the Marburg viral hemorrhagic fever event in Angola, transmission 
within health care settings played a major role on the amplification of the outbreak (WHO, 2009). Nosocomial clustering, with transmission to HCWs, was a prominent feature of severe acute respiratory syndrome (SARS). (Ofner-Agostini et al., 2006 )\& Ho PL, Tang XP, Seto WH.(2003). Similarly, HCWs were infected during the influenza pandemics (Jensen et al, 2005). In some settings (Brazil and Indonesia), more than half the neonates admitted to neonatal units acquired a HCAI, with reported fatality rates between $12 \%$ and 52\%( Allegranzi et al (2008).

\section{Transmission of health care-associated infections}

Transmission of health care-associated pathogens takes place through direct and indirect contact, droplets, air and a common vehicle. Transmission occurs mostly via large droplets, direct contact with infectious material or through contact with inanimate objects contaminated by infectious material. Performance of high-risk patient care procedures and inadequate infection control practices contribute to the risk. Transmission of other viral (e.g. human immunodeficiency virus (HIV), hepatitis B) and bacterial illnesses including tuberculosis to HCWs is also well known. (Jensen et al, 2005). Transmission of health care-associated pathogens from one patient to another via HCWs' hands requires five sequential steps (Girou and Oppein, 2001) these are:

(i) Organisms are present on the patient's skin, or have been shed onto inanimate objects immediately surrounding the patient; Health care associated pathogens can be recovered not only from infected or draining wounds, but also from frequently colonized areas of normal, intact patient skin. Lowbury, 1969) \& Bhalla, Aron and Donskey, 2007 ).

(ii) Organisms must be transferred to the hands of HCWs;

(iii) Organisms must be capable of surviving for at least several minutes on HCWs' hands; Several studies have shown the ability of microorganisms to survive on hands for differing times (Musa \& Desai, 1990).

These studies clearly demonstrate that contaminated hands could be vehicles for the spread of certain viruses and bacteria. Whether care is provided to adults or neonates, both the duration and the type of patient care affect HCWs' hand contamination. HCWs' hands become progressively colonized with commensal flora as well as with potential pathogens during patient care. (Pittet et al., 1999, \& Pessoa-Silva et al, 1999). Bacterial contamination increases linearly over time. Pittet et al, 1999). In the absence of hand hygiene action, the longer the duration of care, the higher the degree of hand contamination. The dynamics of hand contamination are similar on gloved versus ungloved hands; gloves reduce hand contamination, but do not fully protect from acquisition of bacteria during patient care. Therefore, the glove surface is contaminated, making cross-transmission through contaminated gloved hands likely.

(iv) For transmission to occur, hand washing or hand antisepsis by the HCW must be inadequate or entirely omitted, or the agent used for hand hygiene inappropriate. When HCWs fail to clean their hands between patient contact or during the sequence of patient care - in particular when hands move from a microbiologically contaminated body site to a cleaner site in the same patient - microbial transfer is likely to occur (Gupta A et al, 2004). To avoid prolonged hand contamination, it is not only important to perform hand hygiene when indicated, but also to use the appropriate technique and an adequate quantity of the product to cover all skin surfaces for the recommended length of time.

(v) The contaminated hand or hands of the caregiver must come into direct contact with another patient or with an inanimate object that will come into direct contact with the patient. Crosstransmission of organisms occurs through contaminated hands. Factors that influence the transfer of microorganisms from surface to surface and affect cross-contamination rates are the type of organism, source and destination surfaces, moisture level, and size of inoculums (Harrison, 2003) showed that contaminated hands could contaminate a clean paper towel dispenser and vice versa. The transfer rates ranged from $0.01 \%$ to $0.64 \%$ and $12.4 \%$ to $13.1 \%$, respectively. 


\section{Health care associated infection and hand hygiene}

It is an apparent testimonial to its importance that health care associated infection is a significant patient safety hazard and continues to harm patients in the 21st century. Much of this harm can be avoided through better application of measures which already exist, including universal implementation of hand hygiene improvement methods.

There is substantial evidence that hand antisepsis reduces the transmission of health care-associated pathogens and the incidence of HCAI (Boyce and (Pittet, 2002), (Larson, 1988) \& Larson, 1999). Semmelweis demonstrated in 1847 that the mortality rate among mothers delivering at the First Obstetrics Clinic at the General Hospital of Vienna was significantly lower when hospital staff cleaned their hands with an antiseptic agent than when they washed their hands with plain soap and water (Larson, 1988). In the 1960s, a prospective controlled trial compared the impact of no hand washing versus antiseptic hand washing on the acquisition of $S$. Aureus among infants in a hospital nursery (Mortimer, 1962) The investigators demonstrated that infants cared for by nurses who did not wash their hands after handling an index infant colonized with $S$. aureus acquired the organism significantly more often, and more rapidly, than did infants cared for by nurses who used hexachlorophene to clean their hands between infant contacts. This trial provided compelling evidence that compared with no hand washing, hand cleansing with an antiseptic agent between patient contacts reduces transmission of health care associated pathogens.

In addition to these studies, outbreak investigations have suggested an association between infection and understaffing or overcrowding that was consistently linked with poor adherence to hand hygiene (Fridkin et al, 1996) These findings show indirectly that an imbalance between workload and staffing leads to relaxed attention to basic control measures, such as hand hygiene, and spread of microorganisms. Hand hygiene is therefore a core element to protect patients against HCAIs and colonization with multiresistant micro-organisms (Pittet et al, 2006).

\section{Global response}

The World Alliance for Patient Safety is an evolving program of the WHO which was established to raise the profile of patient safety within the global health care agenda. The decision taken in 2004 was to focus the effort and attention of the First Global Patient Safety Challenge on the problem of health care-associated infection. Action on hand hygiene improvement is considered at the core of the First Challenge, with field testing of the WHO implementation strategies developed in conjunction with the WHO Guidelines on Hand Hygiene in Health Care.(WHO, 2008).

The First Global Patient Safety Challenge of the WHO World Alliance for Patient Safety, entitled - Clean Care is Safer Cart has followed a classic approach to health improvement. It called for a concerted global effort to effect policy and intervention strategies to enhance patient safety through implementation of a simple, low-cost health improvement intervention (improved compliance with hand hygiene in health care) to contribute to the prevention of HCAI. To achieve its aims, it has required an action plan for each country, and has involved lobbying for national political action on hand hygiene improvement.

This action positions hand hygiene improvement in health care as a key component of an infection control/quality and safety health improvement program (Runciman \& Moller, 2008). Hand hygiene is considered to be the primary measure necessary for reducing HCAI. Although the action of hand hygiene is simple, the lack of compliance among health-care workers continues to be a problem throughout the world (WHO, 2009)

Principally, the Challenge was conceived to facilitate global awareness-raising about the issue of HCAI and its connection with hygiene and cleanliness in health care settings, focusing attention on the burden and impact of these infections on infection control and patient safety. The implementation of the challenge is built upon four predetermined key success factors. First is to raise worldwide awareness of the impact of healthcare-associated infections on patient safety and promote an exchange of information; second is to build commitment from countries to give priority to reducing 
healthcare-associated infections; third is to issue recommendations bringing the scientific evidence for optimal promotion of hand hygiene and to identify a strategy for their implementation; then lastly is to test the implementation of the hand hygiene recommendations in specific sites worldwide as part of an integrated package of actions in the areas of clean products (blood safety), clean practices (safe clinical procedures), clean equipment (injection and immunization safety), and clean environment (safe water and sanitation in healthcare) (Allegranzia et al, 2007).

\section{Hand Hygiene: Definitions and historical perspective}

The word hygiene is derived from the name Hygeia, who was the Greek goddess of healing (Encyclopedia). In modern usage, hygiene usually refers to cleanliness and especially to any practice which leads to the absence or reduction of harmful infectious agents. The term hand hygiene includes hand washing (washing hands with non-antimicrobial soap), antiseptic hand wash (washing hands with water and soap or another detergent containing an antiseptic agent), antiseptic hand rub (rubbing hands with an antiseptic hand rub) and surgical hand antisepsis (preoperative antiseptic hand wash or Hand rub performed by surgical personnel). (Centres for Disease Control and Prevention, 2002).

Although hand washing has been considered a measure of personal hygiene for centuries, the specific link between hand washing and the spread of infectious diseases has been reported only during the last 200 years (WHO, 2009). In the mid-1800s, studies by Ignaz Semmelweis in Vienna, Austria, and Oliver Wendell Holmes in Boston, USA, established that hospital-acquired diseases were transmitted via the hands of HCWs. Semmelweis recommended that hands be scrubbed in a chlorinated lime solution before every patient contact and particularly after leaving the autopsy room.

The 1980s represented a landmark in the evolution of concepts of hand hygiene in health care. The first national hand hygiene guidelines were published in the 1980s, (Simmons, 1981, Garner et al, 1986 and Bjerke, 2004) followed by several others in more recent years in different countries. In 1995 and 1996, the CDC/Healthcare Infection Control Practices Advisory Committee (HICPAC) in the USA recommended that either antimicrobial soap or a waterless antiseptic agent be used for cleansing hands upon leaving the rooms of patients with multidrug-resistant pathogens ((HICPAC, 1995 and Garner, 1996).

The present WHO guidelines are based on the previous document and represent the most extensive review of the evidence base related to hand hygiene. They guidelines aim to expand the scope of recommendations to a global perspective, foster discussion and expert consultation on controversial issues related to hand hygiene in health care, and to propose a practical approach for successful implementation (WHO, 2009).

\section{Religious and cultural aspects of hand hygiene}

Personal hygiene is a key component of human well-being regardless of religion, culture or place of origin. Human health related behaviour, however, results from the influence of multiple factors affected by the environment, education, and culture. So there are several reasons why religious and cultural issues should be considered when dealing with hand hygiene and planning a strategy to promote it in health-care settings. In the increasingly multicultural, globalized community that is health-care provision today, cultural awareness has never been more crucial for implementing good clinical practice in keeping with scientific developments. Immigration and travel are more common and extensive than ever before as a result of the geopolitically active forces of migration, asylumseeking and, in Europe, the existence of a broad, borderless multistate

Union (WHO, 2009). With the increasingly diverse populations accompanying these changes, very diverse cultural beliefs are also more prevalent than ever. This evolving cultural topography demands new, rapidly acquired knowledge and highly sensitive, informed insights of these differences, not only among patients but also among HCWs who are subject to the same global forces.

It is clear that cultural - and to some extent, religious - factors strongly influence attitudes to inherent community hand washing which, according to behavioural theories are likely to have an impact on compliance with hand cleansing during health care (WHO, 2009). 
Hand hygiene can be practised for hygienic reasons, ritual reasons during religious ceremonies, and symbolic reasons in specific everyday life situations. Islam for example, have precise rules for hand washing included in the holy texts and this practice punctuates several crucial moments of the day (WHO, 2009).

Of the five basic tenets of Islam, observing regular prayer five times daily is one of the most important pillars. Personal cleanliness is paramount to worship in Islam ( Lawrence \& Rozmus, 2001). Muslims must perform methodical ablutions before praying, and clear instructions are given in the Qur'an as to precisely how these should be carried out (Muftic, 1997). The Prophet Mohammed always urged Muslims to wash hands frequently and especially after some clearly defined tasks (Katme, 1999).

Ablutions must be made in freely running (not stagnant) water and involve washing the hands, face, forearms, ears, nose, mouth and feet, three times each. Additionally, hair must be dampened with water. Thus, every observant Muslim is required to maintain scrupulous personal hygiene at five intervals throughout the day, aside from his/her usual routine of bathing as specified in the Qur'an. These habits transcend Muslims of all races, cultures and ages, emphasizing the importance ascribed to correct ablutions. (Ahmed et al, 2006).

In contrast, the Christian faith only specifically requires the ritual sprinkling of holy water on hands before the consecration of bread and wine, and washing of hands after touching the holy oil (the latter only in the Catholic Church). In general, the indications given by Christ's example refer more to spiritual behaviour, but the emphasis on this specific point of view does not imply that personal hygiene and body care are not important in the Christian way of life (Whitby, McLaws \& Ross, 2006).

\section{Variation in Hand Hygiene Compliance}

Multimodal programmes for increasing hand hygiene compliance are now recommended as the most reliable, evidence-based method for ensuring sustainable improvement. (Pittet D et al, 2000). WHO has developed and tested a multimodal Hand Hygiene Improvement Strategy to translate into practice the present guidelines. Unfortunately, compliance with hand hygiene practices has been below an acceptable level or very low in both developed and developing countries. (WHO, 2006). So, improvement of compliance with hand hygiene is needed.

Reasons which explain insufficient compliance or suboptimal ractices are multiple and may vary according to the health care setting and the resources available. The lack of appropriate infrastructure and equipment to enable hand hygiene performance is one of the most important.

The cultural background and even religious beliefs can play an important role in hindering good practices of hand hygiene. Some practitioners see improving compliance as being about changing human behavior and therefore requires inputs from a wide range of specialists including behavioral, psychological and social science. So to improve the compliance rates it is important to follow a planned and step wise approach to the development of interventions, using insight from behavioral science (Bartholomew et al, 2006). The table below outlines the main factors affecting hand hygiene compliance.

Table 1. The main factors affecting compliance with hand hygiene.

\begin{tabular}{|l|l|}
\hline Material Factors & $\begin{array}{l}\text { - Convenient and accessible hand hygiene facilities } \\
\text { e.g. fast-drying hand rubs, no-touch sinks, hand rubs } \\
\text { at patientsbedsides, hand rubs outside patients } \\
\text { rooms, hand rubs on the patients_notes trolley } \\
\text { during a ward round }\end{array}$ \\
- Preparations which do not cause skin irritation
\end{tabular}


Texila International Journal of Clinical Research

Volume 3, Issue 2, Dec 2016

\begin{tabular}{|c|c|}
\hline & - Preparations which are aesthetically acceptable \\
\hline Behavioural and social factors & $\begin{array}{l}\text { - Perceived danger for carer of omitting hand } \\
\text { hygiene } \\
\text { Practices } \\
\text { - Perceived benefit for dependent or patient } \\
\text { - Concern for third party opinion e.g. peer pressure, } \\
\text { conforming to social ideals } \\
\text { - Gender }\end{array}$ \\
\hline Factors in a healthcare institution & $\begin{array}{l}\text { - Avoid overcrowding and understaffing } \\
\text { - Rewards and sanctions } \\
\text { - Promotion of a positive culture for hand hygiene } \\
\text { - Provision of reminders for hand hygiene } \\
\text { - Encourage active participation in the design of } \\
\text { handhygiene programmes at all levels }\end{array}$ \\
\hline
\end{tabular}

Source (WHO, 2009)

Generally compliance rates are very low. It is 38.9 for doctors and 61.4 \%for nurses (Gilbert K, Stafford C., Crosby K., Fleming E. and Robert G, 2010) and it is as high as 75\% for nurse to $47 \%$ ( Randle et al, 2010) and also in another study it is 43.4 for nurses while it is low as $12.3 \%$ for doctors (Batista, 2010). So it is apparent that doctor's compliance is generally less than nurses. It may be that doctors have a distinct culture associated with levels of power which means that they can be difficult with respect to accepting change (Brooks \& Brown, 2003). Historically, doctors have been a powerful pressure group and are perceived as being resistant to change, or opposed to threats to their autonomy (Allegranzi \& Pittet, 2009). Similarly, Ferlie and Shortell state that doctors often resist efforts to standardize practices and impose rules, as in complying with effective hand hygiene (Ferlie \& Shortell, 2001).

It may be that the cultural aspect affects doctorssompliance, especially if they know that they were observed. The same findings regarding physicians were observed by Korniewicz. and El-Masri (2010) that they are less likely to be compliant with hand washing guidelines. This low compliance persisted when examined by external accredited body compared to the other health care workers whereas they showed statistically significant improvement in hand hygiene compliance after the visit (Korniewicz. and El-Masri, 2010). But when comparing different groups of physicians, professors showed the highest compliance rate of $78.4 \%$ while residents and graduation students did not achieve more than 50\% compliance. (Gilbert K, Stafford C., Crosby K., Fleming E. and Robert G, 2010).

One research evaluated hand hygiene compliance by day of the week (Monday through Saturday) and time of observation. Increasingly, during the week the compliance rate (Randle. et al, 2010 ) was highest on Monday (96.5\%) compared to Friday (83.7\%). This means that compliance was better during the first part of the week compared to the end of the week. This also applied to studies in industry, showing a declining work output as the work week progresses; suggesting cumulative fatigue to employee morale as a contributing factor (Duggan J., Hensley S., Khuder S., Papadimos T, and Jacobs L, 2008). The study also revealed that compliance rate is higher when observed in afternoon shifts compared to morning shifts.

These above percentages changed when there is intervention like using bed rail antiseptic system: improved from $36.4 \%$ to $51.5 \%$ but declined six months later (Giannitsioti E. et al, 2009). Although in the same study, the percentage in the already existing bed rail antiseptic system wards did not change. However when using contact precautions also does not alter the percentage of compliance much: for nurses it is $61.4 \%$ for patients in contact precaution rooms compared to $57.1 \%$ in noncontact precaution rooms (Gilbert K, Stafford C., Crosby K., Fleming E. and Robert G, 2010). 
The last findings contradicted what was found in the literature which suggested that hand hygiene compliance is somewhat better for patients in contact precaution rooms.

Another finding was that compliance rate is higher among health care workers after doing any procedure for patients to $72.1 \%$ compared to $41.7 \%$ before doing any procedures. (Korniewicz. and El-Masri, 2010). Although, pre procedure hand hygiene intends to protect the patients against infections and maximize risk reduction, whereas post procedure hand hygiene intends to protect health workers and other patients.

As expected, high risk procedures show better compliance than low risk procedures (Korniewicz. and El-Masri, 2010). Also another study examined the effect of feedback intervention of hand hygiene compliance through a control trial group; unfortunately there was no significant effect of this feedback on improving compliance (Marra et al, 16 2008).

Since complying with hand hygiene has a good correlation with behavioural change, therefore examining the potential determinants of hand hygiene compliance is very important. With help of qualitative study. (Erasmus, Brouwer, Beeck, Oenema, Daha., Richardus and Brug, 2009) examined and analysed the behavioural determinants of hand hygiene compliance among different hospital healthcare workers, including physicians, nurses, and medical students. The hand hygiene behaviour of healthcare workers appeared to be motivated by self-protection and a desire to clean oneself after a task that is perceived to be dirty. Nurses and medical students expressed the importance of hand hygiene for preventing crossinfection among patients and themselves, whereas physicians expressed the importance of hand hygiene but also perceived a lack of evidence for the importance of hand hygiene in preventing cross-infection.

Personal beliefs about the efficacy of hand hygiene and the examples set and norms established by senior staff in a hospital are of major importance for hand hygiene compliance. Medical students tend to copy the hand hygiene behaviour of their superiors, leading to noncompliance when they observe noncompliance by others. Physicians mentioned that their noncompliance was associated with a perceived lack of evidence that hand hygiene is effective in the prevention of hospital-acquired infection, which could be an explanation for the inverse correlation found between the level of education and the rate of hand washing compliance (Erasmus, Brouwer, Beeck, Oenema, Daha., Richardus and Brug, 2009).

\section{WHO hand hygiene multimodal improvement strategy}

Based on the evidence and recommendations from the WHO Guidelines on Hand Hygiene in Health Care, a number of components are needed for an effective multimodal strategy for hand hygiene. The WHO multimodal hand hygiene improvement strategy was proposed to translate into practice the WHO recommendations on hand hygiene and this is accompanied by a wide range of practical tools (WHO, 2009) To facilitate the change, this change project used the WHO Hand Hygiene Implementation multimodal improvement strategy (WHO, 2009).

The five essential elements of the strategy include:

- System change: ensuring that the necessary infrastructure is in place to allow health-care workers to practice hand hygiene. Including infrastructures provision, hand washing facilities, alcohol hand rub dispersion, adequate staffing, supplies provision (water, soap, antiseptic), polices \& guide lines setting

-Training and education: providing training for health-care professionals about the importance of hand hygiene and the correct procedure of hand washing and rubbing

-Evaluation \& feedback: monitoring \& evaluation of the system setting; including monitoring of hand hygiene practices (hand washing, alcohol hand rub use, wearing of gloves and giving performance feedback

-Reminders at the workplace: (e.g. posters) reminding health-care workers about the importance of hand hygiene and about the appropriate indications and procedures for performing it. 
-Institutional safety climate: this includes creation of a hand hygiene safety culture with the participation of both individual HCWs and senior managers. It includes: active participation at institutional \& individual levels, and washing priority —allocating money and time.

\section{Summary}

The literature review highlighted the historical context and the main cultural and professional barriers to hand hygiene. The importance of hand hygiene to patient and HCW safety was highlighted and this was also evidenced by the First Global Patient Safety Challenge. Multi-factorial barriers require evidence based multimodal interventions. The five elements of the WHO Hand Hygiene Multimodal Improvement Strategy were described as the main intervention for this change management project.

\section{Methods}

\section{Introduction}

Change has become an ever-present feature of our work environment, and the management of these continuing changes is a key challenge for all managers. The need for change in the health service is now widely recognised - by public, by professions and by government. As Burnes (2000) rightly claims _what almost everyone would like is a clear and practical change theory which explains what changes organisations need to make and how they should make them'. He goes on to explain that instead what is available is a wide range of confusing and contradictory theories, approaches and recipes. McAuliffe (2000) argues therefore that managers should be prepared to adopt a contingency approach - choosing or developing the model to suit the particular situation. Hence, the change agent's quest — should not be to seek out an all-embracing theory but to understand the strengths and the weaknesses of each approach and the situations in which each can best be applied, according to Burnes (2000).

In this chapter, the steps to implement the selected HSE change model (2009) are described; in particular how the WHO multimodal strategy was implemented in the three ICUs

\section{Change model}

For the change to be successful, the HSE change model will be used to drive the project because according to the model background it has been developed to improve the experience of patients and service users, help staff and teams play a meaningful role in working together to improve services and promote a consistent approach to change across the system (HSE, 2009).

The HSE Change Model pays particular attention to the people and cultural aspects of change. Its main vision is that everybody will have easy access to high quality care and services that they have confidence in and staff are proud to provide. (HSE, 2009). It is built on and reflects several core principles:

- Ensuring that the needs of service users and local communities together with the interests of staff are at the core of the change process

- Building integration and a whole-system approach, focusing on the connections, relationships and dependencies between different parts of the system

- Encouraging collaboration between different agencies, local communities, services, teams, professional groupings, trade unions, and between national, area and local levels

- Promoting active engagement and participation of services users, staff and their representatives in the change process

- $\quad$ Placing a particular emphasis on partnership and team working

- $\quad$ Prioritizing long-term sustainable change and improved organizational effectiveness

- Providing for the transfer of knowledge and skill so that the system equips itself to manage change 
- $\quad$ Promoting processes of organizational learning through regular feedback, measurement and evaluation at all stages of the change journey

- $\quad$ Locating the responsibility to manage change at all levels of the system, individual, group and organizational and at local, area and national levels

\section{Steps of implementation of the HSE Change Model}

The HSE Change Model describes the journey of transformation that enables people to move from the current situation to the desired future, in line with a shared vision for change. Figure 1 outlines the HSE Change Model based on the four stages of the project management lifecycle; which are: initiation stage, planning, implementation and mainstreaming. The steps in adopting the HSE Change Model adoption therefore included these four management stages.

\section{Initiation stage: preparing to lead the change}

This is an early preparation and scoping step that is meant to create readiness and a considered case for change, to establish a sense of shared responsibility, and to scope out a solid foundation for successful change. At this step three meetings and two brain storming sessions were carried out followed by a workshop attended by policy makers in the Ministry of Health, the Oral Health department, stakeholders from the selected ICUs and stakeholders from the other ICUs as observors. Under this preparation phase to lead and drive the change the following was done according to HSE change model:

- Identification of what is driving the need for change and the degree of urgency to introduce the hand rub methods besidse increasing compliance towards hand washing. This involved presenting the rationale (drawn from the literature review) regarding the magnitude of health care associated infection and hand hygiene as the most single measure to reduce infection rates. Furthermore, the baseline data about hand hygiene compliance in the three ICUs when done the previous January was not good. All this directed the attention of workshop participants to agree on the project as a priority as soon as possible.

- Clarification of leadership roles descriptions at different levels was written. An authorized team at the and identification of the key influencers and stakeholders at different levels including the HCWs in the selected ICUs was done. This was done through special committee with my assistance and a clear document stating the responsibilities and job senior management level was assigned with clear mandate throughout the whole project. I was the head of the team and this also included the director of oral health department and a representative from human resource department, finance department, quality and safety department, the three ICU's. The main function of this senior team was to design, manage and lead the change process. Further three supervisors were recruited; one for each ICU. The supervisors acted as mid-level managers for each clinic and they were responsible for the day to day follow up of the change project, reporting to me and communicating with the senior team.

- Assessing the readiness and capacity for receiving and accepting the change from the current state to the expected one at the end of the project through a checklist approach and focus group discussions which revealed the need for assistance and support at the level of the ICUs including availing hand rub and hand washing materials, training and education, and reminder posters.

- Attending to organizational politics: I was fortunate that I had held the senior position of Director General of the State Ministry of Health two years previously. So I fully understood the general managerial situation, the political and power dynamics among the ministry and oral health department leaders, and the cultural background and contextual aspects of the stakeholders within the Ministry. All these served as enabling factors for me for better understanding, influence and resolving the problems during the implementation phase.

- Identifying the leverage points and opportunities for change as follows: 
- Strengths : top leaders and managers commitment in the oral health department, wellstructured department and clear policies and managerial hierarchy at all levels of the department.

- Opportunities: top leaders commitment at the level of the Minister and Director General, the oral directorate received ISO 9001:2008 certificate one year ago, and the possibility of allocating more resources from the Ministry to this project

- Weaknesses: Poor compliance with hand washing as assessed in January and affirmed in the focus group discussion; hence concerted action needed (multimodal) to rectify this situation

- Threats: the very short time of the project and pressure to expand the change project to other ICUs immediately even prior to the evaluation of this pilot.

- Performing an initial assessment of the impact of the change. The project impact statement was prepared (see Table 4).

- Outlining the initial objectives and outcomes for the change which was presented as part of the project proposal and agreed on it through the above meetings and workshop.

- Agreeing on the initial resource requirement motivation (including the newly recruited midlevel supervisors): This was done in a meeting involving the supervisors, doctors and nurses of the three ICUs.

- Outlining the initial business case for change-The full proposal document for this change management project was prepared and presented to senior managers.

Table 2. Project impact statement

\begin{tabular}{|c|c|}
\hline How things are now in relation to the issue & $\begin{array}{l}\text { How things should (ideally) be when the } \\
\text { issue has been addressed }\end{array}$ \\
\hline $\begin{array}{l}\text { Behavioural : } \\
\text { health workers } \\
\text { sometimes: } \\
\text { • Wear gloves without Handwashing } \\
\text { • Do not use alcohol hand Rub } \\
\text { - Do not wash hands between patients }\end{array}$ & $\begin{array}{l}\text { Behavioural : } \\
\text { health workers: } \\
\text { - Use alcohol hand rub frequently } \\
\text { • Wash hands or use alcohol hand rub } \\
\text { before putting on gloves and after taking } \\
\text { off gloves } \\
\text { - Wash hands with soap and water when } \\
\text { visibly dirty or soiled }\end{array}$ \\
\hline $\begin{array}{l}\text { Structural: } \\
\text { Handwashing facilities are usually } \\
\text { available but supplies are not available in } \\
\text { some instances }\end{array}$ & $\begin{array}{l}\text { Clinic managers ensure alcohol hand rub } \\
\text { is available in the selected ICUs } \\
\text { Clinic managers ensure soap and clean } \\
\text { running water are available in the selected } \\
\text { ICUs } \\
\text { New policies and guidelines are in place to } \\
\text { demonstrate responsibilities of health } \\
\text { workers in hand hygiene }\end{array}$ \\
\hline Personal: & Personal : \\
\hline
\end{tabular}




\begin{tabular}{|l|l|}
$\begin{array}{l}\text { My role is currently as an interested } \\
\text { observer, and former Director General } \\
\text { I am motivated to make a difference to } \\
\text { improve compliance }\end{array}$ & $\begin{array}{l}\text { I see my role as an engine to ensure clinic } \\
\text { managers and Directorate Head are } \\
\text { motivated to see the change through Also } \\
\text { as a facilitator to help solve problems as } \\
\text { they arise. } \\
\text { I will continue to work with the } \\
\text { Directorate to ensure the change is } \\
\text { sustained }\end{array}$ \\
\hline $\begin{array}{l}\text { Cultural: } \\
\text { Handwashing is currently perceived as not } \\
\text { important and a waste of time and money } \\
\begin{array}{l}\text { Incorrect perception that wearing gloves } \\
\text { replaces hand hygiene }\end{array}\end{array}$ & $\begin{array}{l}\text { Cultural: } \\
\text { Managers spend time and money on } \\
\text { resources for hand hygiene because } \\
\text { everyone agrees it is a priority and it is } \\
\text { worth the time and cost }\end{array}$ \\
\hline
\end{tabular}

\section{Planning stage}

The purpose of planning was to determine the specific detail of the change and to create support for the change process to ensure that people are joining in a concerted effort, with a clear purpose and create a new future for the organization (HSE) It included the following steps:

- Building commitment: This meant that I needed to increase the commitment to the project across the system. Further activities were done aiming at creating a shared sense of vision towards the proposed change. Without commitment and talking the same language especially at the top level, resistance is expected leading to extreme failure of the project. At this stage of the project the full proposal document was presented to the senior leaders and managers exploring the magnitude of health care associated infection, the need for introducing and implementing hand hygiene programs and the detailed activities in the project. Also these issues were communicated to the mid level managers, selected supervisors and stakeholders at the ICUs. Side meetings, one to ones, and sharing some evidence articles was done to communicate the vision and the change rationale. Furthermore, stakeholders capacity building was one of objectives of this work as discussed in the meetings mentioned above.

- Determining the detail of the change: The current situation in the ICU versus the expected future vision was explored. This included the assessment of the current situation against the future vision for change. Feedback of this analysis was delivered to key stakeholders exploring the gap and describing what is needed materials, appropriate system to support the change and specific details for each of the five elements of the WHO multimodal strategy to be applied in the ICUs. The proposed change was to improve hand hygiene compliance among health care workers in ICU s of UAE. This will be done by increasing compliance to hand washing as such, as well as introducing another simple, quick and effective procedure of hand hygiene which is using alcohol hand rub

- Developing the implementation plan- One of the main steps planning the change is developing and agreeing on the implementation plan. The implementation of the WHO multimodal strategy on hand hygiene included five main steps: system change, training and education of healthcare professionals; monitoring of hand hygiene practices and performance feedback; reminders in the workplace and the creation of a hand hygiene safety culture with the participation of both individual HCWs and senior manager (WHO, 2009). A detailed description of each step was written including the time frame for implementation, and roles/responsibilities for each action.

\section{Implementation stage}

This stage focused on implementing and monitoring the implementation/project plan to ensure that it is meeting its purpose and attending to the factors that will assist longer-term sustainability. As 
stated above in the planning phase, the WHO multimodal strategy hand hygiene was planned to be implemented under the following five elements:

- System change

- Intensive care facilities will have sinks for handwashing available in each clinical setting, complete with access to safe running water, soap and disposable towels.

- Refilled water tank available in each clinic as a backup if piped tap water discontinued

- Alcohol based hand rub available at each point of care in the ICUs according to the WHO standards for antimicrobial efficacy and in sufficient quantities.

- Durable, heavy duty and good quality dispensers purchased

- Supplies of good quality examination and sterile surgical gloves were maintained

- Storage system for the above materials with $25 \%$ stock alarm developed. This software system automatically gives alarm when the stock of the above materials in the ICUs reached $25 \%$ asking the manager in charge to deliver materials to the immediately.

- $\quad$ Providing guidelines and standard operating procedures about hand hygiene including guidelines on when to wash hands and when to use alcohol rub. These included _My 5 moments for hand hygiene' (WHO, 2009).

- Training and education. This is one of the crucial elements of the strategy. Also it remains one of the critical success factors of the project. The objective of the training was stated clearly aiming to induce behavioural and cultural change and ensure that competence is deep-rooted and maintained among all staff in relation to hand hygiene (WHO, 2009). The targeted group were selected including senior staff in the oral health department at the highest level, the supervisors and the doctors at the mid level and finally all the other health care workers. Fifteen training sessions were delivered addressing infection control, definitions and impact of health care associated infection, source and ways of infection transmissions, patient safety in general, WHO programmes of patient safety, WHO programmes on hand hygiene including the WHO multi modal hand hygiene improvement strategy and WHO programmes on My 5 moments for hand hygiene'. Other tools apart from the above mentioned sessions are hand hygiene brochures, training films from WHO website and practical sessions demonstrating how to hand wash and how to hand rub.

- Reminders in the work place: These are important tools to remind health care workers in the ICUs about the importance of hand hygiene, the indications and the procedures of hand washing and hand rub. Also these reminders were used to educate patients and visitors. The reminders used in the ICUs are posters and stickers in the clinic walls and points of care. The posters used as a reminder tool are: WHO My five moments for hand hygiene poster, when and how to hand wash poster, and when and how to hand rub poster.

- Evaluation and feedback: The monitoring \& evaluation (M\&E) system was set up, including monitoring of hand hygiene practices and compliance (hand washing, alcohol hand rub use, gloves wearing), knowledge and perception of health care associated infection and system setting. Also giving performance feedback to the stakeholders is very important. The key success indicators are: increase in hand hygiene compliance, improvement in infection control / hand hygiene infrastructures, increase in usage of hand hygiene products, improved perception of hand hygiene and improved knowledge of hand hygiene (WHO, 2009). The evaluation tools will be detailed further in the next chapter.

- Institutional safety climate: The institutional safety climate refers to creating an environment and the perceptions that facilitate awareness-raising about patient safety issues while guaranteeing consideration of hand hygiene improvement as a high priority at all levels. For this to take place, active participation at both the institutional and individual levels, awareness of individual and institutional capacity to change and improve (self-efficacy), and partnership with patients and patient organizations is needed (HSE project as a precious pilot which finds support from everywhere. Moreover, in one of our regular meeting with doctors when talking about sustainability of hand hygiene practices in their ICUs they said that they cannot imagine that they can stop doing or 
practising it especially after they know that they are at risk of acquiring infection also and not just the patients.

\section{Mainstreaming stage}

The purpose of mainstreaming is to focus attention on the success of the change effort and on integrating and sustaining the new ways of working and behaving. This stage also focuses on mechanisms for evaluation and continuous improvement (HSE, 2009). This stage has two steps:

- Making it the way of our business: The purpose of this step is to help people and stakeholders in the project to integrate and practice hand hygiene new behaviours, skills and practices. This was done by acknowledging success from time to time and motivating staff to do more and more during the process. Also, I used to work with the targeted HCWs personally and give them support on their daily activities especially during the first two weeks so as to overcome any resistance to the change process. My support and work with HCWs in ICUs gave me idea about the process and pathways and clear lines of responsibility and accountability for decision-making which is very important to support the process of change.

- $\quad$ Evaluating and learning: Evaluation has been defined as the systematic and structured process of reviewing an experience, determining its worth (HSE, 2009). The objective of this step is to build a system of continuous evaluation and learning from implementation of the project change process. This will build on the M\&E system devised for the change project. The focus is on improving the organisation's readiness to engage in future change and to discontinue any activity that no longer serves the needs of the new organisational reality (HSE, 2009). This is also relevant to mainstreaming adoption within other ICUs and within other curative settings.

\section{Summary}

This change project applied the WHO Multimodal Strategy elements to the implementation phase. In line with the HSE change management model, specific steps were undertaken to initiate the project, plan the change, implement and secure commitment to mainstreaming. The activities and methods described depended on the support of a range of stakeholders within the oral health department and staff in the ICUs, all keen to undertake this change and adopt change as the way we do our business. My own role in this has been as a leader and initiator of change as well as more practically and personally working alongside HCWs in the selected ICUs. My role continues to sponsor the change and adoption of better compliance to hand hygiene and mainstreaming to other settings.

\section{Evaluation}

\section{Introduction}

Evaluation is defined as a method of measuring the extent to which an intervention achieves its intended objectives. Evaluation also involves making judgements about the value of what is being evaluated (Gerrish and Mawson, 2005).

In the health care context, evaluations are generally undertaken for a clinical intervention, care programme or service innovation and it is recognized that new initiatives in patient care and service delivery should be subject to evaluation in order to judge their effectiveness, efficiency, economy and equity (Gerrish and Mawson, 2005, and Lazenbat, 2002). Evaluation provides practical information to help decide whether a development or service should be continued or not. Evaluation also forms an important part of everyday clinical practice in that care provided to individual patients is evaluated in order to inform clinical decisions about the care of that patient.

An essential component of the change project was its evaluation; and this chapter describes the evaluation tools used, the outcomes of the change including the results of pre and post-assessments, and ending with a short summary of the evaluation. 
Texila International Journal of Clinical Research

Volume 3, Issue 2, Dec 2016

\section{Change Indicators}

This change project was evaluated continuously and the change indicators loosely (Table 5) follow the project impact statement defined from the start

Table.3. Projected outcomes of the change project

\begin{tabular}{|c|c|}
\hline Pre intervention & Post intervention \\
\hline 1. Current compliance with hand hygiene practices & 1. Improved Compliance \\
\hline $\begin{array}{l}\text { 1.1.current hand washing practices } \\
\text { 1.2.current use of alcohol hand rub } \\
\text { 1.3.current gloves wearing practices }\end{array}$ & $\begin{array}{l}\text { 1.1.increase hand washing practices } \\
\text { 1.2. increase use of alcohol hand } \\
\text { rub } \\
\text { 1.3. increased gloves wearing }\end{array}$ \\
\hline 2. Current staff attitude \& perception & $\begin{array}{l}\text { 2. Improved staff attitude } \& \\
\text { perception }\end{array}$ \\
\hline $\begin{array}{l}\text { 2.1.priority to hand washing (time \&money) } \\
\text { 2.2.current culture to using gloves as replacement } \\
\text { for hand wash } \\
\text { 2.3.current culture of risk infection transmission }\end{array}$ & $\begin{array}{l}\text { 2.1. high priority to hand washing } \\
\text { 2.2.gloves not replacement for hand } \\
\text { wash culture } \\
\text { 2.3.improved services quality }\end{array}$ \\
\hline 3. Current services quality & 3. Improved services quality \\
\hline $\begin{array}{l}\text { 3.1.current status of service inputs:- infrastructures } \\
\text { (alcohol hand rub) supplies available } \\
\text { *clean water supply } \\
\text { *soap } \\
\text { *antiseptics staff availability }\end{array}$ & $\begin{array}{l}\text { 3.1.improved services inputs } \\
\text { available hand rub } \\
\text { available supplies } \\
\text { * available water supply } \\
\text { * adequate soap } \\
\text { *adequate antiseptics } \\
\text { available staff }\end{array}$ \\
\hline $\begin{array}{l}\text { 3.2.current status of processes staff training staff } \\
\text { motivation \& active engagement M\&E system } \\
\text { setting new polices \& guide lines setting }\end{array}$ & $\begin{array}{l}\text { 3.2. improved services process } \\
\text { trained personnel motivated \& } \\
\text { engaged staff Active M\&E system } \\
\text { Available policies\& guidelines }\end{array}$ \\
\hline $\begin{array}{l}\text { 3.3.current status of outputs } \\
\text { Patient satisfaction } \\
\text { Infection rate }\end{array}$ & $\begin{array}{l}\text { 3.3.improved services output } \\
\text { Satisfied customers } \\
\text { Reduced infection rate }\end{array}$ \\
\hline
\end{tabular}

\section{Evaluation tools}

Two types of evaluation tools were used:

1) Observation checklist to assess the service and compliance with hand hygiene,

2) Focus group discussions to assess attitude and perceptions of health workers.

\section{HCWs compliance observation check list}

\section{Objectives:}

To assess compliance with the opportunity of hand hygiene by healthcare personnel within 3 ICUs

\section{Methodology:}

- The checklist included facility resources: No of HH sinks, officers/ICUs, chairs, number of health care workers by professional category.

- In addition, observation of compliance of HCWs was done without the knowledge of health care workers (using mystery shopper approach). 
- Observers were trained and conducted pilot observation period with members of the project team

- Direct observation was used to monitor compliance using the three measures from WHO my 5 moments tool that were valid for ICUs; before patient contact, after patient contact and after clinical procedure

- Each opportunity was then coded as to whether the individual complied (took the opportunity to wash hands or use alcohol rub as appropriate)

- Participants (HCWs) were classified as dentist/doctor or nurse

- Data collection took place from 8 am to $2 \mathrm{pm}$ in the morning. On average one clinic observation took about an hour and twenty minutes each

\section{Data analysis:-}

- All observed data was recorded manually on standardized sheets

- X2 tests were used to test whether variation in compliance was statistically significant across HCWs types, ICUs, time of day \& type of opportunity

- Logistic regression model was used to test whether there was a relationship between compliance and reminders after adjustment for other covariance's

- Data analysis was conducted using SPSS version 10 and STATA version 9.

\section{Focus Group discussions}

The focus group discussion (FGD) method is a facilitated interview with several people on specific topic or issue to be explored in depth (Bryman \& Bell, 2007) In this project, perceptions of health workers were assesssed pre $\&$ post project through focus group discussions.

\section{Objectives:}

To understand the cultural and behavioral determinants of hand hygiene among health care workers in the three ICUs.

\section{Methods:}

- Participants: we required 6-8 health care workers representing the two professional categories

- Informed consent was taken from each participant

- An experienced facilitator ran the session with an assistant to record the session and take notes. Participants responses were transcribed and proofed

- A presentation on hand hygiene with a review of definitions \& guidelines for hand hygiene was presented to participants of FGDs

- Is there a relation between not performing hand hygiene and risk of cross-contamination, in what way?

- Is hand hygiene necessary before/after patient contact or any procedures (and why)?

- Is hand hygiene necessary before/after performing any procedures (and why)?

- Is hand hygiene necessary before /after touching patient surrounding (and why)?

- Is hand hygiene necessary after gloves removal (and why)?

- Could gloves be used as replacement of hand hygiene?

- In your opinion, what are possible reasons for non-compliance.

-Probing questions on possible reasons: Poor training: lack of knowledge of the importance of hand hygiene in preventing infections and lack of understanding of the appropriate hand hygiene technique

-Staff shortage, work overload/time constraints

-Absences of institutional commitment to hand hygiene/lack of role model

-Difficult access to points of conventional hand hygiene

-Non-compliance should be aggressed (possible solutions)

- FGDs duration lasted on average about an hour. Each FGDs meeting was followed by a debriefing session among the facilitator and study assistant and myself as project lead.

\section{Outcomes of change}

- The following results were found three weeks into the intervention and 3 weeks post-intervention The change was initiated in March 2011 (baseline). The first assessment of compliance took place 
Texila International Journal of Clinical Research

Volume 3, Issue 2, Dec 2016

three weeks later and the post intervention assessment was undertaken in May 2011. Longer evaluation was not possible due to the short time of the change project

Table 4. Rate of compliance with hand hygiene by healthcare workers

\begin{tabular}{|c|c|c|c|}
\hline \multirow[t]{3}{*}{ Variable } & \multicolumn{3}{|c|}{ Compliance rate } \\
\hline & At base line & $\begin{array}{l}\text { 3weeks into the } \\
\text { intervention }\end{array}$ & $\begin{array}{l}\text { 3weeks post } \\
\text { intervention }\end{array}$ \\
\hline & No (\%) & No (\%) & No (\%) \\
\hline \multicolumn{4}{|l|}{ 1. Intensive care } \\
\hline Medical ICU & $17 / 41(41 \%)$ & $32 / 56(57 \%)$ & 72/76 (97\%) \\
\hline cardiac ICU & $11 / 20(55 \%)$ & $50 / 72(69 \%)$ & 94/100 (96\%) \\
\hline Neonatal ICU & $10 / 25(40 \%)$ & 26/32 (\%81) & $30 / 32(94 \%)$ \\
\hline \multicolumn{4}{|l|}{ 2. Professional Category } \\
\hline Doctors & $14 / 45$ (31\%) & $32 / 70(46 \%)$ & 96/104 (92\%) \\
\hline Nurses & $24 / 41(59 \%)$ & $76 / 90(84 \%)$ & $100 / 104(96 \%)$ \\
\hline \multicolumn{4}{|l|}{ 3. Hand hygiene method } \\
\hline Hand Wash (soap \& water) & $38 / 86(44 \%)$ & $82 / 160(51 \%)$ & 126/208 (61\%) \\
\hline Hand Rub & & 69/160 (43\%) & $181 / 208(87 \%)$ \\
\hline \multicolumn{4}{|l|}{ 4. Hand hygiene Indication } \\
\hline $\begin{array}{l}\text { Before patient touch/any } \\
\text { procedures }\end{array}$ & $17 / 40(43 \%)$ & $54 / 80(68 \%)$ & 99/104 (95\% \\
\hline $\begin{array}{l}\text { After patient touch/any } \\
\text { procedures }\end{array}$ & $21 / 46(46 \%)$ & $54 / 80(68 \%)$ & 97/104 (93\%) \\
\hline Total (overall) & $38 / 86(44 \%)$ & $108 / 160(68 \%)$ & 196/208 (94\%) \\
\hline
\end{tabular}

The hand hygiene compliance of healthcare workers was monitored in the three Intensive Care Areas at base line, at 3weeks after starting the intervention and at 3 weeks post intervention with a total 454 hand hygiene opportunities and over all hand hygiene compliance was found to be $75 \%$.

Hand hygiene showed gradual improvement with time, starting from $44 \%$ at base line compared to $68 \%$ at 3 weeks and $94 \%$ at 3 weeks post intervention successively (Table 6).

\section{Hand hygiene compliance}

The escalating trend of hand hygiene compliance rate across the different assessment periods remained valid for the three ICUs (Figure 2). At base line, the compliance rate for the 3 ICUs ranged between $40-55 \%$, which increased to $57-81 \%$ at 3 weeks after starting the intervention and reached the peak with $94-97 \%$ at 3 weeks post intervention.

The hand hygiene compliance of all ICU s showed statistically significant difference $(\mathrm{p}<0.05)$ between the base line data and3 weeks after dat difference starting. There is also stastically between the 3 weeks and 3 significant first weeks intervention and also between base line and post 3 weeks intervention $(\mathrm{p}<0.05)$ 
There was no statistical difference between ICUs in hand hygiene (Table 5); neither at baseline (41\%, 55\% and 40\%) nor post intervention (97\%, 96\% and 94\%) ( $\mathrm{P}>0.05)$.

Figure.2 Rate of compliance with hand hygiene in Intensive care areas:

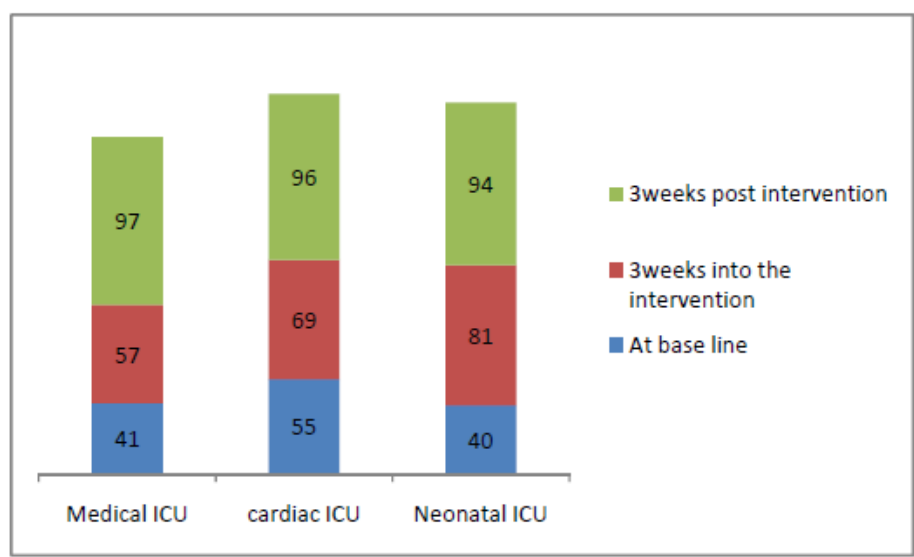

\section{Hand hygiene compliance by professional category}

Compliance with hand hygiene showed statistically significant differences $(\mathrm{P}<0.05)$ between health workers categories at all assessment periods (Figure 3). Nurses scored better compliance (59 \% at base line, $84 \%$ at 3 weeks after starting the intervention and $96 \%$ at 3weeks post intervention assessment), compared to Doctors (31\% at base line, $46 \%$ at 3 weeks after starting the intervention and $94 \%$ at 3 weeks post intervention).

The hand hygiene compliance for both doctors and nurses, showed escalating trends with statistically significance variation $(\mathrm{P}<0.05)$ across the different assessment periods (base line, the first 3 weeks and 3 weeks post intervention).

Figure.3. Hand hygiene compliance by professional category:

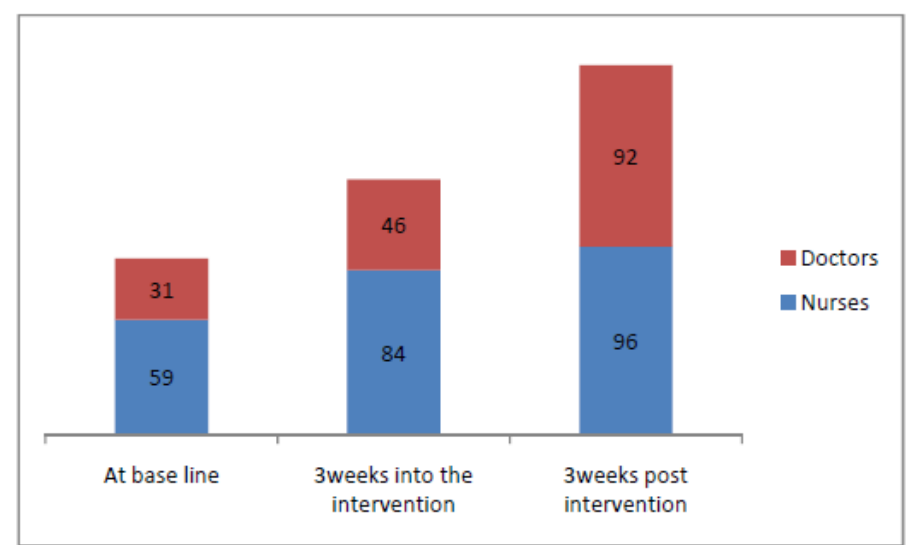

\section{Hand hygiene compliance per hand hygiene method}

The base line assessment of hand hygiene method showed low compliance (44\%) with hand washing, while at that time the alcohol hand rub had not yet been introduced in the health care facilities.

The use of water and soap was the preferred hand hygiene method (51\% compliance) compared to alcohol hand rub (43\% compliance) 3 weeks after starting the intervention (Figure 4). Yet, this preference at 3 weeks post intervention shifted to favor the alcohol hand rub (87\% compliance) compared to water and soap hand washing (69\% compliance). 
The increasing hand hygiene compliance patterns across the different assessment periods showed statistically significant variation $(\mathrm{P}<0.05)$ both for hand washing $(51 \%$ at the first 3 weeks intervention compared to $61 \%$ at 3 weeks post intervention) as well as for alcohol hand rub ( $43 \%$ at the first 3 weeks intervention compared to $87 \%$ at 3 weeks post intervention).

Figure.4. Hand hygiene compliance per hand hygiene method

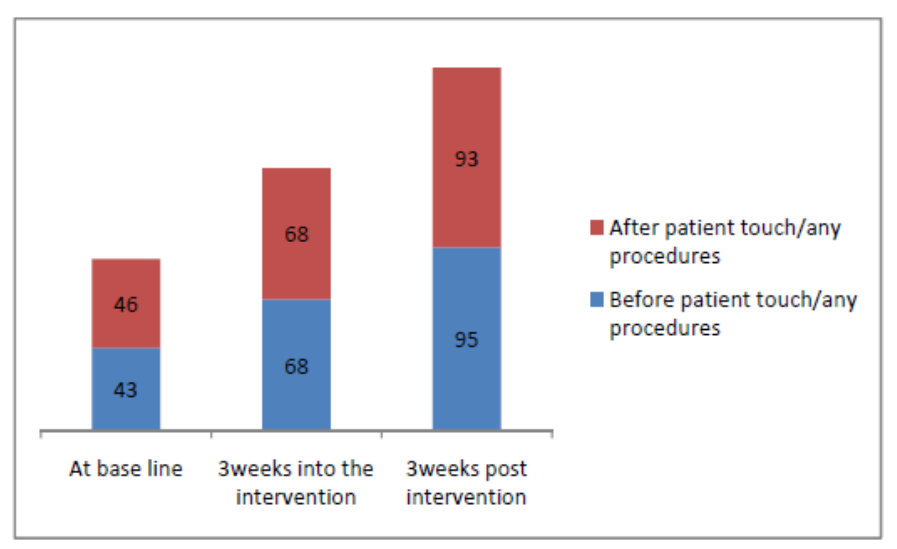

\section{Summary}

Hand hygiene showed rapid improvement from $44 \%$ at base line to $68 \%$ in the first 3 weeks and $94 \% 3$ weeks post intervention. There was no statistically significant difference $(\mathrm{P}>0.05)$ between the 3 ICUs in this respect. There was no difference in hand hygiene before touching patients compared to after patients during all assessment periods.

Compliance to hand hygiene showed statistically significant differences $(\mathrm{P}<0.05)$ between health workers categories in all assessment periods with doctors scoring better compliance compared to nurses. The use of water and soap started off as the preferred method of hand hygiene, yet this preference shifted to favor alcohol hand rub post-intervention at $87 \%$ (compared to water and soap hand washing at $69 \%$ ).

\section{Discussion \& conclusion}

\section{Introduction}

The evaluation demonstrated promising results from the intervention in the three intensive care units. In this chapter, the strengths and limitations of the project are discussed. The implications from this change project for improving hand hygiene are also presented with recommendations for future projects.

\section{Strengths \& limitations of the project:}

Strong leadership commitment was the driving strength in this project both at the level of the Health care Authority and in the Health directorate. Moreover, a well-structured oral health department and clear policies and managerial hierarchy was another strength.

Implementing the change model, with new policies and guidelines and continuous follow up and evaluation mechanisms has proved to be a real challenge due to resistance to change and tolerance to the existing status quo. There was also initial difficulty of communication between management and staff in the selected ICU s, but this was overcome later.

Although more resources were reallocated to this project, still resources are a limiting factor for sustainability. Adding to this shortage of staff especially trained nurses have proved to be real threats to the project implementation. 


\section{Implications of the change project for management}

\section{Overall hand hygiene compliance}

At base line, the overall hand hygiene compliance was found to be below the expectation reporting a rate as low as $44 \%$, which is below the hand hygiene compliance reported in UK study at 2010 by Batista, (55.4\%) and it is also far below hand hygiene compliance reported in studies conducted at Canada and Italy (76\%) (Batista, 2010)

Yet, the data showed considerable and steady progress in overall compliance in the first 3 weeks of intervention with $68 \%$ hand hygiene compliance and 3 weeks post intervention compliance reaching up to $94 \%$. This post intervention compliance level, is far better than the previously mentioned studies.

\section{Hand hygiene compliance per professional category}

The baseline data showed that the hand hygiene compliance of both doctors and nurses were below the expectations at 59\% and $31 \%$. Yet, the post intervention compliance data showed considerable improvement in both doctors and nurses compliance particularly at 3 weeks post intervention (96\% for doctors and $92 \%$ for nurses).

It is well documented in published literature that doctors were found to be more resistant to change and to have poor compliance with infection prevention and control standard (Randel, Clarke \& Storr, 2006) while nurses compliance were known to be higher than doctors (Hugonnet, Prenger \& Pittet, 2002) which could possibly be attributed to doctors'

distinct culture, and their opposing attitude to what they consider as a threat to their autonomy (Randle, Arthur \& Vaughar, 2010). Yet, on the contrary to the above mentioned literature, doctors compliance was found to be better than that of nurses at base line (59\% compared to 31\%), at the first 3 weeks of intervention ( $84 \%$ compared to $46 \%$ ), and at 3 weeks post intervention ( $96 \%$ compared to $92 \%)$.

\section{Hand hygiene compliance per hand hygiene method}

Batista et al 2010, found that hand washing method for hand hygiene was the preferred method in comparison to alcohol hand rub. Yet, the 3 weeks post intervention data showed a different picture as the preference shifted to favor the alcohol hand rub method (87\%) compared to hand wash method (69\%).

\section{Hand hygiene compliance per hand hygiene indication}

It is documented in the published evidence that healthcare workers tend to wash their hands according to their needs (Btista et all, 2010) and thus they tend to comply more with hand hygiene after patient contact compared with their compliance before patient contact (Randle, Arthur \& Vaughar, 2010). Yet, the hand hygiene compliance at all assessment periods (base line, the first 3 weeks of intervention and 3weeks post intervention) showed no statistical difference for opportunities before patient contact compared to those after patient contact.

\section{Recommendations for future improvement}

1. Since the implementation of intervention proved to be beneficial, then the institutionalization and systematic \& sustainable application of these changes might have its rewards in increasing hand hygiene compliance and reduce nosocomial infection transmission in other healthcare settings.

2. Nurses were found to have less compliance in comparison to doctors, the adoption of intervention \& programs specially targeting such an important professional category might provide a good chance of success to increase hand hygiene compliance in health care facilities

3. The systematic and wide application of alcohol hand rub should be encouraged and nested at the top policy makers priority list 
Texila International Journal of Clinical Research

Volume 3, Issue 2, Dec 2016

4. Further research should be adopted to investigate the hand hygiene determinants and to verify the observed association between the implemented interventions and the improvement of hand hygiene compliance in other intensive care areas and other settings.

\section{Reflections on the project}

During the implementation of the project and while I am introducing alcohol hand rub, I faced with strong resistant in one of the clinic. So I decide to pay more attention to healthcare workers in this ICU s by increase training, education and more supervision. Later I discovered that the doctor in charge in the clinic has his own religious concern about using alcohol. I know that Alcohol use is prohibited in Islam or considered an offence requiring a penance because it is considered to cause mental impairment. But that if you drink it or used it in any type of food. This concern took a lot of time from me to conscience him and delayed the intervention for some time in that clinic.

\section{Conclusion}

The hand hygiene compliance rate showed gradual improvement over time, starting from below standard hand hygiene at base line assessment (44\%), to reach its maximum at 3 weeks post intervention assessment (94\%). The introduction of alcohol hand rub was particularly successful and it became the preferred hand hygiene method. These results indicate that the systematic application of the change model and WHO multimodal strategies is associated with improvement in hand hygiene compliance of $l$ healthcare workers and thus it could result in a sustained positive impact. Further work is needed to understand and improve the poorer compliance of doctors compared to nurses.

\section{References}

[1] Ahmed QA et al. (2006). Muslim health-care workers and alcohol based handrubs. Lancet, 367:10251027.

[2] Allegranzi B et al (2008). The burden of hospital-acquired infections (HAI) in developing countries: a systematic review. Poster presented at: 48th Interscience Conference on Antimicrobial Agents and Chemotherapy/46th Annual Meeting of the Infectious Diseases Society of America, Washington, DC, abstr. K4106

[3] Allegranzia B., Storra J., Dziekana G., Leotsakosb A., Donaldsonb L., Pitteta D (2007). The First Global Patient Safety Challenge - Clean Care is Safer Care: from launch to current progress and achievements. Journal of Hospital Infection: 65(S2) 115-123.

[4] Barbacombe J., (2004). Back to the basics - hand washing. Geriatric Nursing 25, 90-92.

[5] Bartholomew LK, Parcel GS, Kok G, Gottlieb NH. Planning health promotion programs: an intervention mapping approach. San Francisco, CA: Jossey-Bass, 2006.

[6] Bhalla A, Aron DC, Donskey CJ (2007). Staphylococcus aureus intestinal colonization is associated with increased frequency of S. aureus on skin of hospitalized patients. BMC Infectious Diseases, 7:105.

[7] Bischoff W, Reynolds T, Scssler C, Edmond M, Wenzel R.,(2000). Hand washing compliance by health care workers: the impact of introducing an accessible, alcohol-based hand antiseptic. Arch Intern Med ; 160:

1017-21 washing compliance by health care workers: the impact of introducing

[8] Bjerke NB., 2004. The evolution: hand washing to hand hygiene guidance.

[9] Critical Care Nursing Quarterly, 27:295-307.

[10] Boyce JM, Pittet D. (2002). Guideline for hand hygiene in healthcare settings. Recommendations of the Healthcare Infection Control Practices Advisory Committee and the HICPAC/ SHEA/APIC/IDSA Hand Hygiene Task Force. Morbidity and Mortality Weekly Report, 51:1-45.

[11] Brooks I, Brown RB., (2003). The role of the ritualistic ceremonial in removing barriers Between subcultures in the National Health Service. J AdvNurs; 38: 341e352.

[12] Burnes, B. (2000). Managing Change. A Strategic Approach to Organisational Dynamics, 3rd Edition. Essex, England: Prentice Hall. 
[13] Centres for Disease Control and Prevention, (2002). Guideline for Hand Hygiene in Health-Care settings: Recommendations of the Healthcare Infection Control Practices Advisory Committee and the HICPAC/SHEA/APIC/IDSA Hand Hygiene task Force. MMWR ; 51 (No. RR-16):1—48.

[14] Cosgrove SE (2006). The relationship between antimicrobial resistance and patient outcomes: mortality, length of hospital stay, and health care costs. Clin Infect Dis ; 42(Suppl. 2):S82eS89.

[15] Duggan J., Hensley S., Khuder S., Papadimos T. And Jacobs L.(2oo8). Inverse correlation between level of professional education and rate of hand washing in a teaching hospital. Infection Control and Hospital Epidemiology vol; 29, no. 6:534-538.

[16] Dumpis U et al (2003). Prevalence of nosocomial infections in two Latvian hospitals. Eurosurveillance, 8:73-78.

[17] Encyclopaedia Britannica Online. www.britannica.com.

[18] Erasmus V., Brouwer W., Beeck, F., Oenema A., Daha J., Richardus H., Brug J., 21 ( 2009). A Qualitative Exploration of Reasons for Poor Hand Hygiene. Among hospital workers: Lack of Positive Role Models and of Convincing Evidence that Hand Hygiene Prevent Cross Infection. Infection Control and Hospital Epedimiology30:415-419

[19] Ferlie E, Shortell S., 2001. Improving the quality of health care in the United Kingdom and the United States: a framework for change. Milbank Q;79:281e315.

[20] Fridkin S et al.(1996): The role of understaffing in central venous catheter- associated bloodstream infections. Infection Control and Hospital Epidemiology, 17:150-158. 